\title{
Implementing small scale processes at the soil-plant interface - the role of root architectures for calculating root water uptake profiles
}

\author{
C. L. Schneider ${ }^{1}$, S. Attinger ${ }^{1,2}$, J.-O. Delfs ${ }^{3}$, and A. Hildebrandt ${ }^{1}$ \\ ${ }^{1}$ Helmholtz Centre for Environmental Research - UFZ, Department of Computational Hydrosystems, Leipzig, Germany \\ ${ }^{2}$ Institute for Geosciences, University of Jena, Jena, Germany \\ ${ }^{3}$ Helmholtz Centre for Environmental Research - UFZ, Department of Environmental Informatics, Leipzig, Germany
}

Received: 19 May 2009 - Published in Hydrol. Earth Syst. Sci. Discuss.: 12 June 2009

Revised: 20 January 2010 - Accepted: 24 January 2010 - Published: 12 February 2010

\begin{abstract}
In this paper, we present a stand alone root water uptake model called aRoot, which calculates the sink term for any bulk soil water flow model taking into account water flow within and around a root network. The boundary conditions for the model are the atmospheric water demand and the bulk soil water content. The variable determining the plant regulation for water uptake is the soil water potential at the soil-root interface. In the current version, we present an implementation of aRoot coupled to a 3-D Richards model. The coupled model is applied to investigate the role of root architecture on the spatial distribution of root water uptake. For this, we modeled root water uptake for an ensemble (50 realizations) of root systems generated for the same species (one month old Sorghum). The investigation was divided into two Scenarios for aRoot, one with comparatively high (A) and one with low (B) root radial resistance. We compared the results of both aRoot Scenarios with root water uptake calculated using the traditional Feddes model. The vertical rooting density profiles of the generated root systems were similar. In contrast the vertical water uptake profiles differed considerably between individuals, and more so for Scenario B than A. Also, limitation of water uptake occurred at different bulk soil moisture for different modeled individuals, in particular for Scenario A. Moreover, the aRoot model simulations show a redistribution of water uptake from more densely to less densely rooted layers with time. This behavior is in agreement with observation, but was not reproduced by the Feddes model.
\end{abstract}

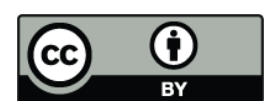

Correspondence to: C. L. Schneider (christoph.schneider@ufz.de)

\section{Introduction}

The global water and carbon cycles are key issues in climate and global change research. Within these complex systems, plants are the central interface between the atmosphere and hydrosphere. Transpiration plays a crucial role for the surface energy balance as well as for the water cycle. It is also linked to the carbon cycle through its close connection with photosynthesis. Hydrological as well as climate models will benefit from an improved understanding of the process of water flow through plants, in particular because they are sensitive to root water uptake parameters (Desborough, 1997; Zeng et al., 1998). Also, great uncertainty in modeling transpiration stems from lack of knowledge about how much water is available to plant roots (Lai and Katul, 2000; Feddes et al., 2001).

Plant water uptake responds to soil moisture limitation at different time and space scales. At the seasonal time scale, plants may adapt their rooting system by root growth, in order to reach moister soil areas (Wan et al., 2002). But also at smaller time scales (like hours to days), plants have been observed to change their uptake zone, and without altering their root system (Sharp and Davies, 1985; Green and Clothier, 1995; Garrigues et al., 2006).

However, models for describing water flow at the soilplant-atmosphere-interface (SVAT-schemes) include these processes only partially. These schemes use a heuristic parametrization for root water uptake that is applied as a sink in the one-dimensional Richards Equation. Commonly, vertical root water uptake profiles are related to the product of a water stress function and the vertical rooting density distribution (like in Feddes et al., 1976). However, this parametrization leads to early predictions of limited transpiration, when densely rooted soil layers dry out (Feddes et al., 2001) and thus neglects the plants adaptive response to water stress.

Published by Copernicus Publications on behalf of the European Geosciences Union. 
In order to deal with these shortcomings, several algorithms have been developed to allow for a longer period of transpiration in a SVAT context. Li et al. (2001) and Teuling et al. (2006) presented models that compensate water stress in one part of the root zone by increased uptake from other soil areas without altering rooting density profiles. Šimůnek and Hopmans (2009) followed a similar approach. They proposed a root water uptake compensation mechanism for HYDRUS (Šimůnek et al., 2006, 2008), which allows for parallel consideration of compensated water and nutrient uptake in three dimensions. Also, besides compensation effects, another mechanism sustaining transpiration in dry soil, is hydraulic redistribution. It is defined as water transfer from wetter into drier soil areas, via flow through the root system. Recently, Siqueira et al. (2008) and Amenu and Kumar (2008) investigated this effect for delayed onset of water stress in a root water uptake model, again based on rooting density profiles.

The above models treat uptake and adaptation in a lumped way, and therefore do not consider the mechanisms at the scale at which they take place. Models which include more detail could be used to gain the necessary process understanding, in order to transfer it to the SVAT scale. Small scale processes of root water uptake have already been implemented in models of varying levels of complexity.

First level models distribute the transpirational demand on the soil domain simply by the spatial distribution of roots either in one (as SVAT models do), two or three dimensions (Vrugt et al., 2001; Clausnitzer and Hopmans, 1994).

Second level models include a description of microscopic water flow along the potential gradient between the soil and the root, either using an effective resistance along this gradient like Gardner $(1960,1964)$ or more realistic radial dependent soil hydraulic properties (Tuzet et al., 2003; de Jong van Lier et al., 2006). The latter cover the nonlinear behavior of unsaturated water flow. This is an important mechanism in drying soils (Schröder et al., 2008), because steep potential gradients develop around the roots. These models can be extended to include root radial resistance additionally to soil resistance (Siqueira et al., 2008; Schymanski et al., 2008). For example Levin et al. (2007) showed with such a combined model that vertical uptake profiles changed depending on the assumed radial resistance. Schymanski et al. (2008) applied such a model to modify root distribution within biological constraints according to soil water availability.

The approaches above imply that the potential on the side of the root is constant throughout the root system. However, Zwieniecki et al. (2003) suggested in a combined measurement and model study that internal gradients along the root xylem exist. Depending on the ratio between the roots radial and axial resistance, the active uptake region could extend over the entire root or just part of it. This research was conducted only for a single root, but might also be relevant for uptake along the entire root system.
Third level models combine a variable xylem potential distribution along the root structure with the flow processes in the soil domain. One such model was introduced by Doussan et al. (1998). Such root water uptake models can be coupled to three dimensional soil water flow models as done in Doussan et al. (2006) or Javaux et al. (2008). Simulations with these detailed models show that the region of water uptake moves with time to deeper and moister layers, when top layers dry out. The coupling of soil and root water flow in the vicinity of the root segments was first based on an averaging approach. A finer spatial discretization of the numerical soil grid around the roots (as shown in Schröder et al., 2009) can represent the local gradients in soil water potential but at the cost of increased computational burden.

In summary, previous research using small scale models for water uptake indicates that both water flow in the soil near the root, but also within the root system itself shape the uptake behavior of the plant. Plant root systems vary greatly in form and morphology, not only between species, but also between individuals of the same species. This chapter contributes to answer the question, how does this variety influence the expected uptake pattern. Therefore, we propose a simplified third level model called aRoot and apply it to simulate the water uptake of an ensemble of root systems of the same species and age. Our model results suggest that water uptake profiles vary significantly between individuals.

\section{Models and methods}

The major assumption for this study is that the process of plant water uptake is gradient driven by the difference between soil water potential and atmospheric demand. In real plants, this leads to a distribution of water potentials from the leaves (stomata control) over the trunks to the stem and finally to the root system. Hence, the outer boundaries of the plants water uptake system are the atmospheric water deficit and the soil water potential. In this model exercise, we only consider the part from the soil up to the root collar. Within this study we make a comparison between two model approaches for root water uptake. One approach uses a full 3-D Richards Equation (see Sect. 2.1) coupled to the Feddes reduction function (Sect. 2.4) to simulate soil water stress effects on root water uptake. The other approach again uses the 3-D Richards Equation to model the bulk soil water flow combined with a smaller scale water uptake model called "aRoot" (Sect. 2.2). This "aRoot" model was divided into two scenarios of different root hydraulic parameterizations.

\subsection{Bulk water flow in the unsaturated zone}

The Richards' equation describing the water movement in the soil system is known as

$\frac{\partial \theta}{\partial t}=\nabla\left[K \nabla\left(\psi_{\text {soil }}+z\right)\right]-\varrho(x, y, z, t)$, 
where $\theta\left[\mathrm{m}^{3} \mathrm{~m}^{-3}\right]$ is the volumetric soil water content, $\varrho\left[\mathrm{m}^{3} \mathrm{~m}^{-3} \mathrm{~s}^{-1}\right]$ is the sink term rate delivered by the root water uptake model (see Eq. 22) for the aRoot approach of volumetric flow rates) and $t[\mathrm{~s}]$ is time. The numerical solution of the Richards Equation for bulk soil water flow is provided by GeoSys (Kolditz et al., 2008).

Volumetric soil water saturation $\theta$ is defined as a function of the soil water potential $\psi_{\text {soil }}[\mathrm{m}]$ and can be expressed by the Mualem-van-Genuchten parametrization (van Genuchten, 1980) as

$\frac{\theta-\theta_{\mathrm{r}}}{\phi-\theta_{\mathrm{r}}}=\Theta=\left[\frac{1}{1+\left|\alpha_{\mathrm{G}} \psi_{\text {soil }}\right|^{n_{\mathrm{G}}}}\right]^{m_{\mathrm{G}}}$,

where $\Theta$ is the normalized (or relative) water content, $\phi$ is the porosity of the soil and $\theta_{\mathrm{r}}$ the residual volumetric water content (at so-called permanent wilting point), where $\alpha_{\mathrm{G}}, n_{\mathrm{G}}$ and $m_{\mathrm{G}}$ are soil specific parameters (see Table 1). $K\left[\mathrm{~m} \mathrm{~s}^{-1}\right]$ in terms of normalized (or relative) water content $\Theta$ is then given by

$K(\Theta)=K_{\mathrm{s}} k(\Theta)=K_{\mathrm{s}} \Theta^{\lambda_{\mathrm{G}}}\left(1-\left(1-\Theta^{\frac{1}{m_{\mathrm{G}}}}\right)^{m_{\mathrm{G}}}\right)^{2}$,

where $\Theta$ can be replaced by $\psi_{\text {soil }}$ using Eq.(2). The saturated hydraulic conductivity $K_{\mathrm{S}}$ as well as the bulk soil porosity $\phi$ are given in Table 1. Accounting for the effect of root segments exploring a certain soil volume, within our model the porosity $\phi$ of all soil grid cells is decreased by the corresponding fraction of volumetric root content. This is motivated by the fact that as root volume increases in a certain soil volume, the soils pore space gets less and hence less water can be hold in this soil volume.

\subsection{The hydraulic root water uptake model "aRoot"}

In the following, we present a stand alone root water uptake model called aRoot, which calculates the sink term for the bulk soil water flow model. Since we apply an analytical expression for the radial water flow towards the root, our model concept does not require intense iteration between the bulk water flow model and aRoot for each time step.

\subsubsection{Water flow within the root system}

Water flow within the plants takes place as a flow from root surface to the inner root xylem (radial) and along the xylem tubes (axial). The hydraulic uptake model applied to the root system is spatially explicit consisting of a network of root segments. Each individual root segment is modeled as a series of axial and radial resistances similar to Doussan et al. (1998). These root resistances operate as an effective value for the underlying processes, like xylem development for the axial pathway and radial connectivity within the root cortex and epidermis (as described in Steudle and Peterson (1998) as the apoplastic, symplastic and transcellular pathways).
Table 1. Model parameters.

\begin{tabular}{|c|c|c|c|c|}
\hline \multicolumn{5}{|c|}{ Root properties } \\
\hline \multirow{2}{*}{$\begin{array}{l}\text { Segm. } \\
\text { Order }\end{array}$} & \multirow[t]{2}{*}{$r_{0}[\mathrm{~m}]$} & \multicolumn{2}{|c|}{$\zeta_{p}[\mathrm{~s}]$} & \multirow{2}{*}{$\begin{array}{l}\mathcal{R}_{l}\left[\mathrm{~s} \mathrm{~m}^{-3}\right] \\
\text { Scenario A/B }\end{array}$} \\
\hline & & Scenario A & Scenario B & \\
\hline 0 & $0.006-0.004$ & $5 \times 10^{10}$ & $5 \times 10^{10}$ & $1 \times 10^{9}$ \\
\hline 1 & $0.004-0.003$ & $1.5 \times 10^{10}$ & $1.5 \times 10^{10}$ & $2 \times 10^{9}$ \\
\hline 2 & $0.003-0.002$ & $7 \times 10^{9}$ & $9 \times 10^{8}$ & $6 \times 10^{9}$ \\
\hline 3 & $0.002-0.001$ & $3 \times 10^{9}$ & $5 \times 10^{8}$ & $8 \times 10^{10}$ \\
\hline $4(\geqq)$ & $0.001-0.0005$ & $1 \times 10^{9}$ & $1 \times 10^{8}$ & $1 \times 10^{12}$ \\
\hline \multicolumn{5}{|c|}{ Soil properties } \\
\hline$\theta_{\text {soil }}^{\text {init }}$ & 0.4 & \multicolumn{3}{|c|}{ initial soil water status $[-]$} \\
\hline$\theta_{\text {soil }}^{\text {PWP }}$ & 0.08 & \multicolumn{3}{|c|}{ permanent wilting point saturation $[-]$} \\
\hline \multicolumn{5}{|c|}{ van Genuchten parameters for sandy soil } \\
\hline$K_{\mathrm{S}}$ & 1.785 & \multicolumn{3}{|c|}{ saturated soil water conductivity $\left[\mu \mathrm{m} \mathrm{s}^{-1}\right]$} \\
\hline$\phi$ & 0.46 & \multicolumn{3}{|c|}{ soil porosity [-] } \\
\hline$\alpha_{\mathrm{G}}$ & 1.44 & \multicolumn{3}{|l|}{$[1 \mathrm{~m}]$} \\
\hline$\lambda_{\mathrm{G}}$ & -0.215 & \multicolumn{3}{|l|}{$[-]$} \\
\hline$\beta_{\mathrm{G}}$ & 0.534 & \multicolumn{3}{|l|}{$[-]$} \\
\hline$m_{\mathrm{G}}$ & 0.348 & \multicolumn{3}{|c|}{$=\beta_{\mathrm{G}} /\left(1+\beta_{\mathrm{G}}\right)$} \\
\hline$n_{\mathrm{G}}$ & 1.534 & \multicolumn{3}{|l|}{$=\beta_{\mathrm{G}}+1$} \\
\hline \multicolumn{5}{|c|}{ Feddes model: water stress function $\beta_{\mathrm{rw}}$ for sandy soil } \\
\hline$\psi_{1}$ & -1 & \multicolumn{3}{|l|}{$[\mathrm{m}]$} \\
\hline$\psi_{2}$ & -2 & \multicolumn{3}{|l|}{$[\mathrm{m}]$} \\
\hline$\psi_{3}$ & -100 & \multicolumn{3}{|l|}{ [m] } \\
\hline$\psi_{4}$ & -150 & \multicolumn{3}{|l|}{$[\mathrm{m}]$} \\
\hline \multicolumn{5}{|c|}{ Boundary conditions } \\
\hline$T_{\text {Pot }}$ & -0.8 & \multirow{2}{*}{\multicolumn{3}{|c|}{$\begin{array}{l}\text { potential transpiration rate }\left[\mathrm{mm}^{3} \mathrm{~s}^{-1}\right] \\
\text { critical xylem water potential }[\mathrm{m}]\end{array}$}} \\
\hline$\psi_{\text {xylem }}^{\text {crit }}$ & -150 & & & \\
\hline
\end{tabular}

Root hydraulic properties are assigned to each root segment according to their root order given by RootTyp (see Sect. 2.3 and Table 1). The axial resistance $R_{\mathrm{ax}}$ is calculated by multiplying the axial resistivity per length with the corresponding root length $l_{\mathrm{r}}$, while the radial resistance $R_{\mathrm{r}}$ is estimated by dividing the specific radial resistivity (material property of each root segment) by root surface area.

The influence of osmotic potential differences are neglected as well as the effect of aquaporins changing the specific radial resistivity per root segment (Steudle, 2000) or the effect of cavitation on xylem vulnerability increasing the axial resistance (Sperry et al., 2003).

For each root segment $n$ the axial flux is implemented by the formula

$J_{\mathrm{ax}}^{n}=\frac{1}{R_{\mathrm{ax}}^{n}}\left(\Delta \psi_{\mathrm{xylem}}^{n}+\Delta z^{n}\right)$

where $\Delta$ is the potential gradient along the root xylem axis between two root nodes. The radial flux, which is the inflow 
from the soil to the root segment $n$ is given by

$J_{\text {rad }}^{n}=\frac{1}{R_{\mathrm{r}}^{n}}\left(\psi_{\text {xylem }}^{n}-\psi_{\text {soil }}^{n}\left(r_{0}\right)\right)$,

with $\psi_{\text {xylem }}^{n}$ denoting the xylem water potential within root segment $n$ and $\psi_{\text {soil }}^{n}\left(r_{0}\right)$ the soil water potential at the root surface of the corresponding soil disc $n$.

By applying the Kirchhoff's Law for summing up all inand outflows at a root node, we receive a system of equations describing the water fluxes of the root network that can be best described in matrix notation such as

$\mathbf{A} \psi_{\text {xylem }}=\mathbf{B} \psi_{\text {soil }}\left(r_{0}\right)+\boldsymbol{c}$,

where $\mathbf{A}$ is the system matrix (regarding radial and axial root resistances) coupling root xylem pressure for interlinked root nodes, $\mathbf{B}$ is the input matrix connecting xylem potential to corresponding soil potentials and $\boldsymbol{c}$ is the offset vector accounting for gravitation (lifting water up over the vertical axis) and the upper boundary condition (flux or potential boundary at root collar). The boundary condition at the root collar is initially fixed to a given flux $T_{\text {Pot }}$. If the corresponding variable collar potential drops below a critical value $\psi_{\text {xylem }}^{\text {crit }}$, then boundary switches to a potential condition and transpirational flux becomes variant.

Rearranging Eq. (6) gives

$\psi_{\text {xylem }}=\mathbf{A}^{-1} \mathbf{B} \psi_{\text {soil }}\left(r_{0}\right)+\mathbf{A}^{-1} \boldsymbol{c}$.

By rewriting Eq. (5) for all root segments $N$ and introducing the conductance matrix $\kappa_{\mathrm{r}}$ (main diagonal matrix containing the inverse of the radial resistances $\boldsymbol{\kappa}_{\mathrm{r}}=$ $\left.\operatorname{diag}\left[1 / R_{\mathrm{r}}^{0}, \ldots, 1 / R_{\mathrm{r}}^{n}, \ldots, 1 / R_{\mathrm{r}}^{N}\right]\right)$ as well as new notations $\mathbf{E}=\mathbf{A}^{-1} \mathbf{B}$ and $\mathbf{d}=\mathbf{A}^{-1} \boldsymbol{c}$ leads to a system of equations for the overall radial fluxes in the root system, namely

$J_{\text {rad }}=\boldsymbol{\kappa}_{\mathrm{r}}\left[(\mathbf{E}-\mathbf{I}) \psi_{\text {soil }}\left(r_{0}\right)+\mathbf{d}\right]$,

where $\mathbf{I}$ is the identity matrix of dimension $N$, the overall number of root nodes. This system can be simplified to

$J_{\text {rad }}=\mathbf{W} \psi_{\text {soil }}\left(r_{0}\right)+\omega$,

where $\mathbf{W}=\boldsymbol{\kappa}_{\mathrm{r}}(\mathbf{E}-\mathbf{I})$ and $\omega=\boldsymbol{\kappa}_{\mathrm{r}} \mathbf{d}$.

\subsubsection{The microscopic radial water flow within the soil}

The microscopic flow towards the root is assumed to be only one dimensional in radial direction towards the root, where the soil domain is modeled as a cylinder of radius $r_{\text {disc }}$ and height $l_{r}$. Local hydraulic gradients in soil water potential towards the root can be obtained with an approximated analytical solution of the Richards equation (steady rate assumption after Jacobsen (1974) and De Willigen and van Noordwijk (1987) where the temporal change in water content is assumed to be $r$ independent)

$\frac{\partial \theta_{\text {soil }}}{\partial t}=\frac{1}{r} \frac{\partial}{\partial r}\left[K\left(\psi_{\text {soil }}\right) r \frac{\partial \psi_{\text {soil }}}{\partial r}\right]=$ const.
In matric flux potential notation, this equation becomes an ODE as

$\frac{1}{r} \frac{\partial \Phi_{\text {soil }}}{\partial r}+\frac{\partial^{2} \Phi_{\text {soil }}}{\partial r^{2}}=$ const. ,

with the following solution

$\Phi_{\text {soil }}(r)=\frac{\tau_{3}}{4} r^{2}+\tau_{2} \log (r)+\tau_{1}$,

where $\tau_{p}$ are integration constants set by boundary/initial conditions.

The matric flux potential $\Phi_{\text {soil }}\left[\mathrm{m}^{2} \mathrm{~s}^{-1}\right]$ is defined as a function of soil water potential $\psi_{\text {soil }}$ by

$\Phi_{\text {soil }}\left(\psi_{\text {soil }}\right)-\Phi_{\text {soil }}^{\text {ref }}=\int_{\psi_{\text {soil }}^{\text {ref }}}^{\psi_{\text {soil }}} K\left(h_{\text {soil }}^{\prime}\right) d h_{\text {soil }}^{\prime}$,

where $\Phi_{\text {soil }}^{\text {ref }}$ and $\psi_{\text {soil }}^{\text {ref }}$ are reference states of the system. For $\psi_{\text {soil }}^{\text {ref }} \rightarrow-\infty$, the reference matric flux potential tends to $\Phi_{\text {soil }}^{\text {ref }} \rightarrow 0$, so

$\Phi_{\text {soil }}\left(\psi_{\text {soil }}\right)=\int_{-\infty}^{\psi_{\text {soil }}} K\left(h_{\text {soil }}^{\prime}\right) d h_{\text {soil }}^{\prime}$.

The solution of this integral depends on the functional form of $K\left(\psi_{\text {soil }}\right)$. Unfortunately, for the Mualem-vanGenuchten parameterization used in our soil water model, no explicit solution is known. Therefore, a closed analytical relationship between water potential $h$ and matric flux potential $\Phi$ cannot be established. Nevertheless, within a certain range of $h$, the matric flux potential can be approximated by the following transfer function

$\Phi_{\text {soil }}\left(r_{0}\right)=b_{1} \exp \left(b_{2}\left|\psi_{\text {soil }}\right|^{b_{3}}+b_{4}\right)$,

with $b_{k}$ soil dependent fitting parameters. For our simulations, the soil parameters $b_{k}$ of Eq. (15) were fitted to the numerical calculated $\Phi$ - $h$-profile for a sandy soil set up by the Mualem-van-Genuchten parameters given in Table 1.

The solution of Eq. 11 (similar to de Jong van Lier et al., 2008 or Schröder et al., 2009) with given boundary conditions (zero flux at outer boundary, radial flux $J_{\text {rad }}$ at inner boundary and a given bulk matric flux potential at a certain radial distance $r_{\Phi_{b}}$ ) can be written as

$\Phi\left(r_{0}\right)=\Phi_{b}+\frac{J_{\mathrm{rad}}}{2 \pi l}\left(\frac{a^{2}-\gamma+\gamma \log \left(a^{2} \gamma\right)}{2-2 \gamma}\right)$,

with $\gamma=\left(r_{\Phi_{b}} / r_{0}\right)^{2}, r_{0}$ the root radius, $r_{\text {disc }}$ the soil disc radius and $r_{\Phi_{b}}=a r_{\text {disc }}$, where $a=0.607$ is proposed by de Jong van Lier et al. (2006). The soil disc radius $r_{\text {disc }}$ is linked to the root length in a given soil volume and is set equal for all root segments $n$ within this volume. 
Hence, the soil water flow corresponding to all root segments is given by the gradient in matric flux potential between the soil-root interface and the bulk soil multiplied with a function determined by the boundary conditions and hence depending on the segment geometry (given by Eq.16),

$J_{\text {rad }}^{n}=g^{n}\left(\Phi_{\text {soil }}^{n}\left(r_{0}\right)-\Phi_{b}^{n}\right)$,

with

$g^{n}=\frac{4 \pi l^{n}\left(1-\gamma^{n}\right)}{a^{2}-\gamma^{n}+\gamma^{n} \log \left(a^{2} \gamma^{n}\right)}$.

Writing the radial soil water flow in matrix notation for all $N$ segments with $\mathbf{G}$ the main diagonal matrix containing the functional terms $g^{n}\left(\mathbf{G}=\operatorname{diag}\left[g^{0}, . ., g^{n}, . ., g^{N}\right]\right.$, we receive

$J_{\mathrm{rad}}=\mathbf{G}\left(\Phi_{\mathrm{soil}}\left(r_{0}\right)-\Phi_{b}\right)$

\subsubsection{Coupling the root and radial soil water flow}

The radial root water flow (9) and the radial soil water flow (19) are set equal (coupled directly via flux type condition)

$\mathbf{W} \psi_{\text {soil }}\left(r_{0}\right)+\boldsymbol{\omega}=\mathbf{G}\left(\Phi_{\text {soil }}\left(r_{0}\right)-\Phi_{b}\right)$,

with $\Phi$ given as a nonlinear function of $h$ depending on soil parameters (here given by Eq. (15)) resulting in

$\mathbf{W} \psi_{\text {soil }}\left(r_{0}\right)+\boldsymbol{\omega}=\mathbf{G}\left(f\left(\psi_{\text {soil }}\left(r_{0}\right)\right)-f\left(\psi_{\mathrm{b}}\right)\right)$.

This nonlinear system of equations is solved based on a certain bulk water potential and the given root system with its specific boundary condition at the root collar (forming the matrices $\mathbf{W}, \mathbf{G}$ and the vector $\boldsymbol{\omega}$ ) leading to a distribution of the water potential at the soil-root-interface $\psi_{\text {soil }}\left(r_{0}\right)$.

\subsubsection{The sink term for the macroscopic bulk water flow in the unsaturated zone}

Figure 1 shows the model scheme we use to implement the sink terms into the bulk soil water flow model and how the bulk soil water potential feeds back to the microscale radial soil water flow model. Our concept underlies the assumption that all soil discs around root segments covering a certain soil volume $\Omega_{i j k}$ share uniform bulk water potential $\psi_{b}$ and soil disc radii $r_{\text {disc }}$.

The sink term $S$ for the bulk soil water flow model is calculated by summing up the radial fluxes $J_{\text {rad }}^{m}$ of all soil discs $m$ belonging to a certain bulk soil volume $\Omega_{i j k}$ as

$$
\begin{aligned}
S(i, j, k)= & \sum_{m} J_{\text {rad }}^{m} \quad \forall J_{\text {rad }}^{m} \in \Omega_{i j k} \\
\Omega_{i j k}= & \left\{(x, y, z) \in \mathbb{R}^{3}: a_{i} \leq x \leq a_{i+1},\right. \\
& \left.b_{j} \leq y \leq b_{j+1}, c_{k} \leq z \leq c_{k+1}\right\},
\end{aligned}
$$

with $i, j \in\left\{1 \ldots N_{\text {hor }}+1\right\} \subset \mathbb{Z}, k \in\left\{1 \ldots N_{\text {vert }}+1\right\} \subset \mathbb{Z}$, where $N_{\text {hor }}$ and $N_{\text {vert }}$ are the number of bulk soil volumes in the

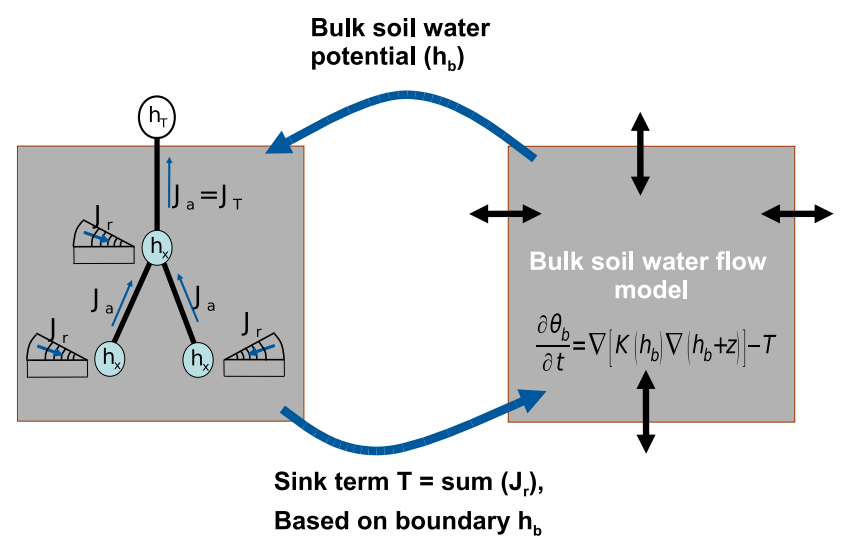

Fig. 1. Concept of coupling microscale radial flow to bulk flow including xylem potentials for a bulk soil volume $\Omega_{i j k}$.

horizontal and vertical direction and the rules for $a_{i}, b_{j}$ and $c_{k}$ are the following

$$
\begin{aligned}
a_{i} & =x_{\min }+(i-1) \Delta x ; \Delta x=\frac{x_{\max }-x_{\min }}{N_{\mathrm{hor}}} ; \\
b_{j} & =y_{\min }+(j-1) \Delta y ; \Delta y=\frac{y_{\max }-y_{\min }}{N_{\mathrm{hor}}} ; \\
c_{k} & =z_{\min }+(k-1) \Delta z ; \Delta z=\frac{z_{\max }-z_{\min }}{N_{\mathrm{vert}}} .
\end{aligned}
$$

\subsection{The root architecture model}

The root architecture model used for our simulations is based on the generic model RootTyp by Pagès et al. (2004). The generator creates realizations of the same species by simulating growth as a random process covering root emission, axial and radial growth, sequential branching, reiteration, transition, decay and abscission. The interplay of these processes is parameterized plant specifically. We used a parameter set for plant species of sorghum type, which is a class of numerous grass species. The size of the root system depends on the stage of plant development, hence age. All generated root systems are characterized by their interconnected root segments of a designated order. The order defines the segments axial resistance per length (due to alternating xylem vessel elaboration), specific radial resistivity (due to different stages of suberization) and root radius (see Table 1). Figure 2 shows exemplary a root system for one of the 50 realizations.

\subsection{The Feddes model}

The RWU function of Feddes (like in Feddes et al., 2001) is the following

$\varrho(h(x, y, z))=\beta_{\mathrm{rw}}(h) \frac{L_{V}^{a}(x, y, z)}{\int_{V} L_{V}^{a}(x, y, z) d V} T_{\mathrm{Pot}}$,

with $L_{V}^{a}\left[\mathrm{~m} \mathrm{~m}^{-3}\right]$ the accumulated root length per volume (RLD) at a point, $V$ the overall volume of the soil-root domain and $T_{\text {Pot }}\left[\mathrm{m}^{3} \mathrm{~s}^{-1}\right]$ the potential transpiration flow 

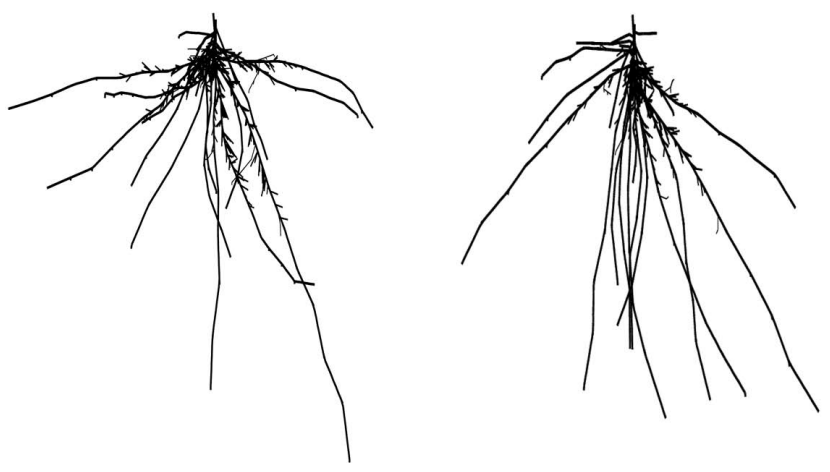

Fig. 2. 2-D-plot of two arbitrarily chosen root system realizations created by the root architecture generator RootTyp.

rate. To get the volumetric flow rate $S(x, y, z)$, the extraction rate (of volume of water per volume of soil per time) $\varrho\left[\mathrm{m}^{3} \mathrm{~m}^{-3} \mathrm{~s}^{-1}\right]$ has to be applied to a specific soil volume $\Omega$.

The Feddes approach includes a water stress function $\beta_{\mathrm{rw}}$, where the most common implemented stress function has the form shown in Fig. 3.

\subsection{Model input and scenarios}

The model exercise was divided into three characteristic cases: (1) the Feddes approach widely applied in current SVAT models based on the RLD neglecting the root systems network character as well as microscopic radial water flow within the soil, the aRoot simulations for (2) Scenario A where higher order roots have higher radial resistances and the aRoot simulations for (3) Scenario B where higher order roots have lower radial resistances (see Table 1). The reason for dividing the aRoot model in two Scenarios (A and B) is the ongoing debate on the range of the radial resistance values (references from Steudle and Peterson, 1998; Zwieniecki et al., 2003).

We performed the simulations for all three cases on 50 root system realizations. The simulation time for root water uptake for all realizations was set to 10 days (with time steps of $\Delta t=30 \mathrm{~min}$.) starting from a uniform, initial saturation of $\Theta=0.4$. The bulk soil water flow model runs on a $2.5 \times$ $2.5 \times 2.5[\mathrm{~cm}]$ grid cell size. The overall soil domain size in $x$-, $y$ - and $z$-direction is $27.5 \times 27.5 \times 22.5[\mathrm{~cm}]$ among all root realizations. The plants root system age was set to 1 month (28 days) where there was no further root growth applied within the simulation time.

The transpiration rate was assumed to be time invariant with $T_{\mathrm{Pot}}=-8 \times 10^{-10} \mathrm{~m}^{3} \mathrm{~s}^{-1}$ over the 10 days of unlimited uptake, as long as the root collar potential has not exceeded a given threshold. If the corresponding variable collar potential drops below this critical value $\psi_{\text {xylem }}^{\text {crit }}$, then the boundary switches from a flux type to a potential type condition and transpirational flux gets variant.

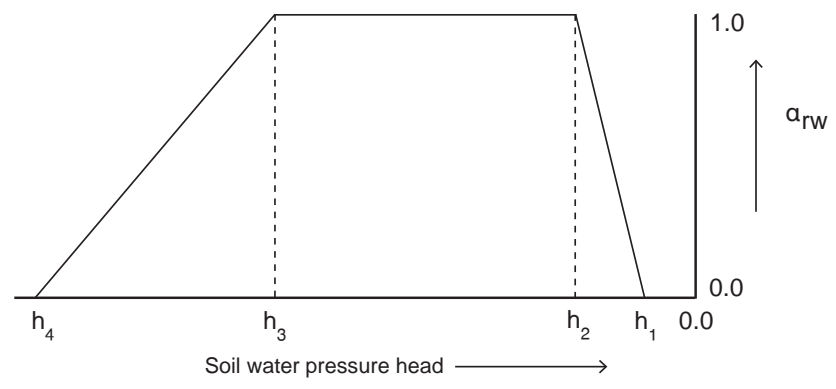

Fig. 3. Water stress function used in the Feddes model: Water uptake above $h_{1}$ and below $h_{4}$ is set to zero due to oxygen deficit and wilting point. Between $h_{2}$ and $h_{3}$ water uptake is maximal $\left(\alpha_{\mathrm{rw}}=1\right)$. Above $h_{2}$ and below $h_{3}$, the so-called critical point, water uptake gets limited where the precise value of $h_{3}$ is assumed to vary with potential transpiration rate $T_{\mathrm{Pot}}$.

The specific radial resistance $\zeta_{p}$ (as a material constant for root order $k$ with a given thickness of the roots radial pathway) is assumed to decline with increasing $k$ caused by less suberization, where $\zeta_{p}$ is calculated by multiplying the materials resistivity $\chi_{p r}$ with the roots radial thickness $r_{c}$. Radial resistance $R_{\mathrm{r}}$ is the ratio of $\zeta_{p}$ to the root outer surface area $\left(R_{\mathrm{r}}=\zeta_{p} /\left(2 \pi r_{0} l\right)\left[\mathrm{s} \mathrm{m}^{-2}\right]\right)$. Also, we assume that axial resistance per length $\mathcal{R}_{l}$ increases with root order (due to decreasing root radius), multiplied by the root segment length $l_{\mathrm{r}}$ it gives the axial resistance $R_{\mathrm{ax}}=\mathcal{R}_{l} \times l_{\mathrm{r}}\left[\mathrm{s} \mathrm{m}^{-2}\right]$.

Parameters of Scenario A are in agreement with measurements by Steudle and Peterson (1998)(page 778): Root properties of segment order 2 are referenced by the mature late metaxylem measurements whereas for root order 4 characteristics are given by the early metaxylem. For Scenario B radial resistance was decreased, but only for higher order roots, so that $R_{\mathrm{ax}} / R_{\mathrm{r}}$ is in the range of 0.025 in accordance to the results of Zwieniecki et al. (2003).

\section{Results}

\subsection{Influence of root architecture and hydraulic root parameters on root water uptake behavior}

Figure 4 shows the modeled root water uptake (RWU) versus root length density (RLD). The plotted points represent entities on the bulk scale where the RLD was calculated by counting root segment lengths in each bulk soil grid cells and RWU is the given sink term of the bulk soil water flow in Eq. (1). We plotted all model runs (50 realizations of each, the Feddes approach, aRoot Scenario A and aRoot Scenario B) at three different time steps ( 0,5 and 10 days).

For the initial time step plot (Fig. 4a), all model runs provided very similar results. The results of the Feddes approach match perfectly the 1:1 line which was expected from the model assumption. For later time steps (Fig. $4 \mathrm{~b}$ and c), we 


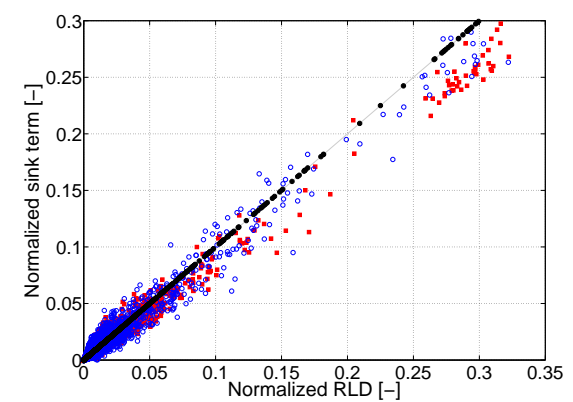

(a) 0 days

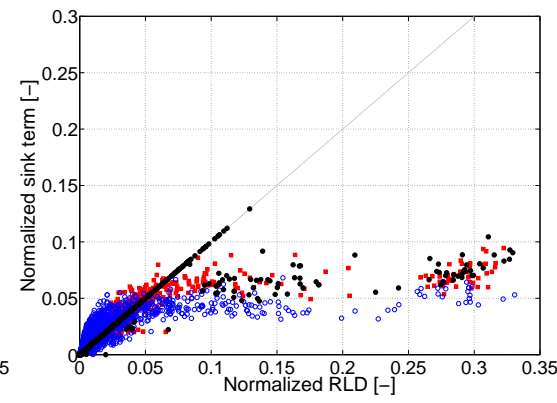

(b) 5 days

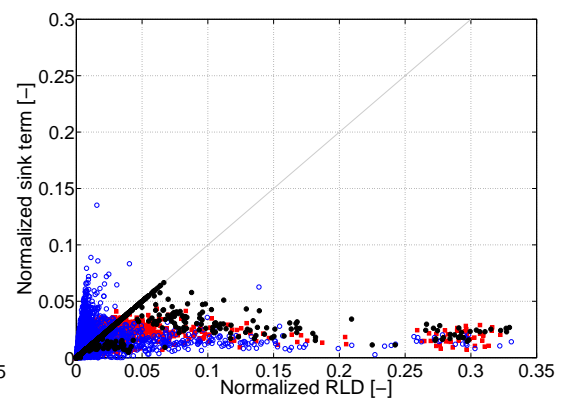

(c) 10 days

Fig. 4. Sink term vs. RLD for 50 Realizations of Scenario A (red square), Scenario B (blue circle) and Feddes (black dot) at (a) initial time step $t=0$, (b) after 5 days and (c) after 10 days (sink terms are normalized by the potential transpiration rate $T_{\mathrm{Pot}}$ and RLD by total root length)

see that Scenarios A and B of aRoot show some compensation effects: water uptake from areas of higher RLD is decreased and this decline is compensated by increased uptake from lower RLD regions where Scenario B shows a stronger compensation than Scenario A does. Also, at $t=5$ and $t=10$ days, the sink terms of the Feddes approach and the aRoot Scenarios A and B were comparably similar for higher RLD (between 0.1 and 0.35). Within the range of lower RLD (normalized values from 0 to 0.2 ), water uptake was highest for the Feddes model and lowest for Scenario B. However, in the part of lower RLD (up to 0.1) the sink terms for the Feddes model remained mostly at the $1: 1$ line with no compensational effects. This missing effects are a straight result of the Feddes model assumptions.

\subsection{Influence of root architecture on vertical uptake profiles}

In Fig. 7, we plotted the vertical profiles for RLD and RWU. For this, both variables were averaged over the horizontal soil domain and normalized by the total root length respectively the potential transpiration rate $T_{\text {Pot }}$.

All 50 root system realizations showed a similar RLD profile resulting in a narrow $90 \%$ confidence band. For the aRoot Scenarios A and B, the RWU profiles showed larger confidence bands than the RLD profile. Moreover, during the simulation, the confidence intervals for the water uptake profiles increased in all three cases. The strongest spread could be seen for Scenario B, while the Feddes approach showed only very little variation.

At the initial time step, $t=0$ days, the mean water uptake profile for both aRoot Scenarios was in the range of the mean RLD profile. The confidence bands showed a slightly higher spread for the uptake profiles than for the RLD profiles. At $t=10$ days, the mean uptake at layers with high RLD was for Scenario B only $40 \%$ of what would be expected by the RLD profile. At the same time, it was up to $300 \%$ higher than RLD at deeper soil layers of lower rooting density. The same trends were observed for Scenario A but with smaller differences between vertical RWU and RLD because of already limited uptake.

Furthermore, the vertical water uptake profiles of Scenarios A and B showed a moving uptake front from layers of high RLD to layers of lower RLD for both scenarios. This shift was faster for Scenario B than for A. Also for Scenario A, RWU was limited earlier than for Scenario B resulting in a slighter compensation of decreased uptake from higher layers (already drier) by increased uptake from lower rooted layers (still wet).

Compared to the aRoot model, we see important differences in the Feddes model: at timestep $t=0$ days the profiles of vertical uptake do perfectly match the RLD profiles as can already be seen in Fig. 4a. With time the uptake in the layers of higher RLD decreases but with no compensation of water uptake from less densely rooted layers. The width of the confidence bands remains almost constant in the layers of decreased uptake while they still match the RLD profiles in the nonlimited deeper layers. This general uptake behavior leads to early limitation of water uptake compared to the aRoot model.

\subsection{Influence of root architecture on critical point of water uptake limitation}

Another important factor for modeling root water uptake is the relation between transpirational demand and resulting collar potential (or vice versa). This can only be investigated with a model where xylem potentials are resolved, which is the case for aRoot but not for the Feddes model.

Figure 5 shows the evolution of the root collar potentials over simulation time for all 50 realizations. The influence of root radial resistance on collar potential becomes obvious by comparing Fig. 5a (Scenario A) and b (Scenario B). We see that plants in Scenario A would exhibit a more negative xylem pressure than in Scenario B. This is due to the larger resistance in the flow path from soil to xylem. The curves 


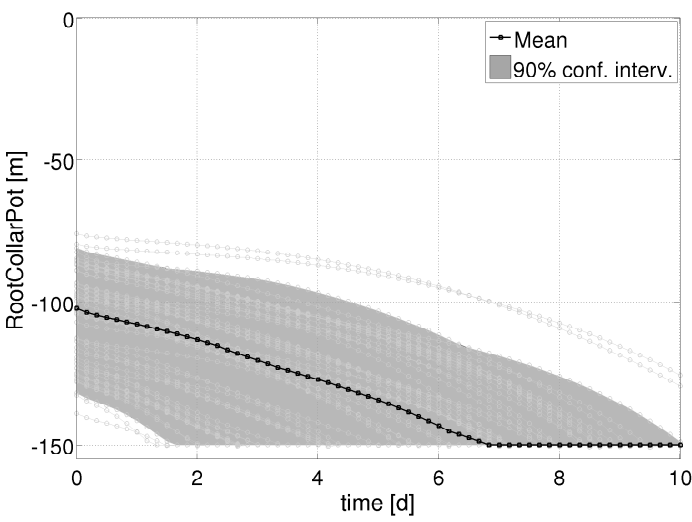

(a) Scenario A

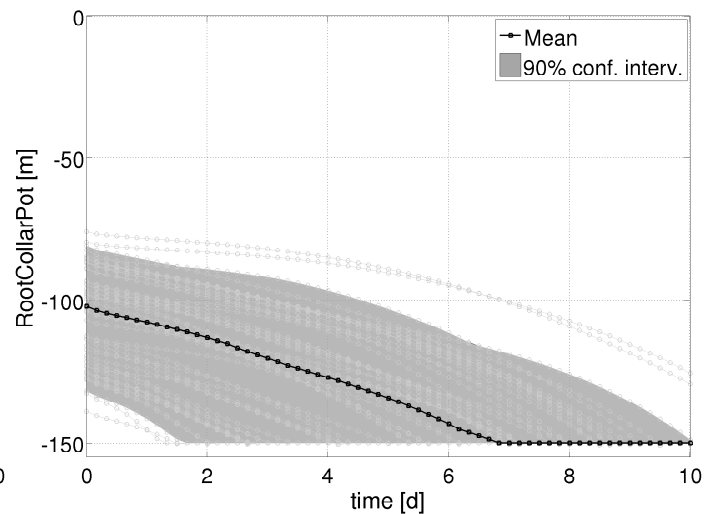

(b) Scenario B

Fig. 5. Temporal evolution of collar potentials for (a) Scenario A and (b) Scenario B. The black dotted line is the mean xylem water potential at the root collar for all 50 realizations. The gray band denotes the $90 \%$ confidence interval and the light gray lines are the individual collar potential curves.

also show a high variability among the realizations for Scenario A where for $\mathrm{B}$, the confidence interval is narrow for most of the simulation. We also see that plants in Scenario A reach the critical point of limited water uptake much earlier than in Scenario B. There, water uptake is still unlimited at the end of the 10 day long simulations for all realizations.

In Fig. 6, we plotted only for Scenario A mean soil saturation versus resulting actual transpiration. We observed a wide spread of expected water uptake from individual root architectures. While in early limited root systems uptake was reduced by $40 \%$, other systems were still not limited after 10 days of transpiration.

\section{Discussion}

In this model exercise we generated 50 root architectures using the model RootTyp of Pagès (Pagès et al., 2004). These realizations could be interpreted as 50 different individuals of the same plant species and age. The obtained root systems show similar root length density profiles, as indicated by the narrow confidence intervals shown in Fig. 7. Root length density decreases exponentially with depth for all individuals. This is in accordance to observations not only for grasses, but for all biomes (Schenk and Jackson, 2002).

For these root systems, root water uptake was simulated over 10 days of transpiration by three model cases: the architecture based aRoot model by Scenarios A and B and the root length based SVAT approach by Feddes. We implemented Scenarios A and B both based on current literature in plant physiology (see Steudle and Peterson, 1998; Zwieniecki et al., 2003). For Scenario A, the specific radial resistivity of higher order roots is set within the higher range, where for Scenario B it is at the lower limit. The model results for both Scenarios differ, but both show a confidence spread over all modeled individuals, either regarding the

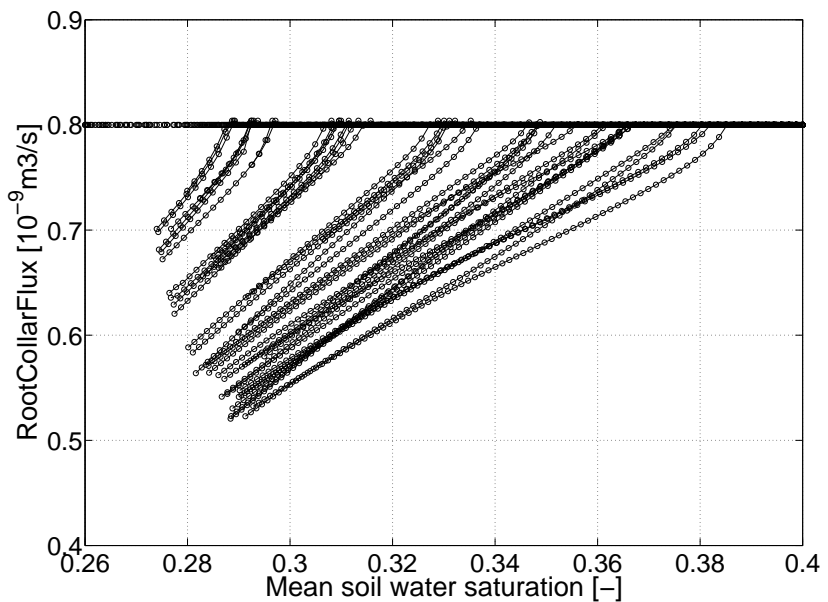

Fig. 6. Individual collar fluxes (black dotted line) for all 50 realizations of Scenario A over mean soil saturation defined as the integral of the entire soil domain (regarding the soil domain as a simple bucket).

evolved collar potential and reaching limiting soil water conditions (Scenario A) or regarding the distribution of vertical uptake profiles over soil depth (Scenario B).

While Scenario A gives vertical uptake profiles that do differ less among the 50 realizations than Scenario B, it shows a high variability in xylem potentials that need to be applied at the root collar. The temporal evolution of collar potential differs among the realizations for Scenario A already at early times, which emphasizes the role of higher root radial resistances. The opposite holds for Scenario B: We see more scattering among the vertical uptake profiles than for Scenario A but less scatter in the evolution of root collar potentials. This variability in the vertical RWU profiles is due to the effects of local soil water depletion. Thus, the influence of root architecture on RWU is either more on the plants side 

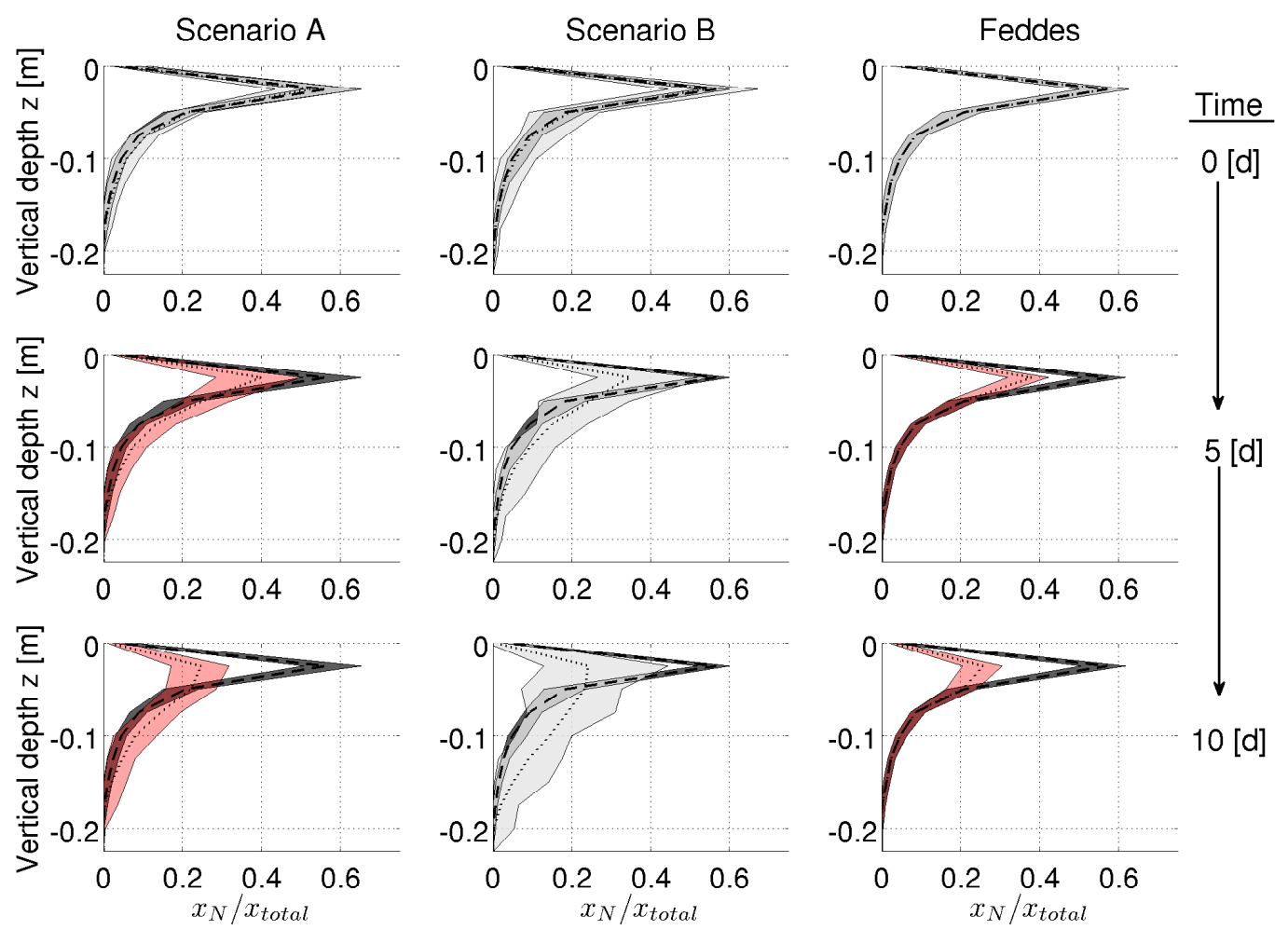

Fig. 7. Vertical Profiles of RLD (dashed) and RWU (dotted) over soil depth for 50 Realizations of Scenario A (left), B (middle) and Feddes (right) at time steps $t=0$ (up), 5 (middle) and 10 (bottom) days. The dark gray band is the 90\% confidence interval for the vertical RLD profile, where the light gray band is the $90 \%$ confidence interval for the RWU profile (transparent red bands denote limited water uptake).

(concerning the temporal evolution of collar potentials, Scenario A) or on the soils side (concerning the vertical uptake profiles, Scenario B).

In our aRoot simulations the modeled root water uptake moves from densely to less densely rooted layers with time. This is in agreement with observation (Garrigues et al., 2006; Lai and Katul, 2000) as well as with results from detailed 3D models for root water uptake (Doussan et al., 2006; Javaux et al., 2008). Our results suggest that the dynamic of this shift depends on the individual root architecture as well as on root properties (here the range of radial resistances). The Feddes approach does not show this moving uptake behavior (as the model does not consider such effects) and additionally lacks the architecture based scattering in water uptake rates versus RLD. Javaux et al. (2008) already pointed out, the parameterization of the Feddes model seems to have little biophysical basis. Our results support this interpretation.

Our simulations show that the occurrence of decreasing water uptake is not at a unique critical point in soil water potential (corresponding to point $\psi_{3}$ in Fig. 3). This was the case, although we used the same soil environment and same plant species (with similar RLD profiles). Rather, this study shows that root architecture influences the critical point of bulk soil water content where water uptake becomes limiting. The diverse access of the root systems hydraulic active roots to the soil water storage explains this model result.
The proposed model aRoot underlies certain assumptions or simplifications. Schröder et al. (2008) has shown, that the local soil hydraulic conductivity drop around the roots becomes important when increasing the size of the bulk soil grid cells. We accounted for this by implementing a microscale radial flow model coupled to the bulk soil water flow. In their model study, Schröder et al. (2009) concluded that for coarser soil discretization, separating the microscale (radial) flow from bulk soil water flow as done in aRoot (similar to their method C) gave the best results compared to fine discretized RWU models. The assumption of uniform bulk water content and soil disc radii for all soil discs covering a certain soil volume is discussed in de Jong van Lier et al. (2006). Further work would be necessary to quantify the influence of this assumption.

Further on, within the current model version of aRoot no root growth occurs within the 10 days long simulation. Although we have not implemented root growth, our simulations can be regarded as a stepwise analysis of water uptake related to a certain soil water distribution. Coupling root growth to soil water availability would change the focus of this chapter from the role of root architecture on RWU to adaptivity issues. Nevertheless, root growth can be implemented into aRoot later. 
Table 2. List of Variables and Abbreviations.

\begin{tabular}{lll}
\hline Symbol & Units & Description \\
\hline$r$ & $\mathrm{~m}$ & radial distance \\
$x, y, z$ & $\mathrm{~m}$ & cartesian coordinates \\
$l_{\mathrm{r}}$ & $\mathrm{m}$ & root segment length \\
$t$ & $\mathrm{~s}$ & time \\
$\psi$ & $\mathrm{m}$ & matric potential \\
$\Phi$ & $\mathrm{m}^{2} \mathrm{~s}^{-1}$ & matric flux potential \\
$\theta$ & $\mathrm{m}^{3} \mathrm{~m}^{-3}$ & volumetric water content \\
$J, T, S$ & $\mathrm{~m}^{3} \mathrm{~s}^{-1}$ & volumetric flow rates \\
$K$ & $\mathrm{~m} \mathrm{~s}^{-1}$ & hydraulic conductivity \\
$R$ & $\mathrm{~s} \mathrm{~m}^{-2}$ & hydraulic resistance \\
$\kappa$ & $\mathrm{m}^{2} \mathrm{~s}^{-1}$ & hydraulic conductance \\
$L_{V}^{a}$ & {$\left[\mathrm{~m} \mathrm{~m}^{-3}\right]$} & accumulated root length per volume (RLD) \\
$\mathrm{RLD}$ & & root length density \\
$\mathrm{RWU}$ & & root water uptake \\
\hline
\end{tabular}

\section{Conclusions}

In this chapter we developed a simplified model, that captures small scale features of plant-water uptake but is still computationally fast. Although our model currently runs with a 3-D Richards Model it is intended for later implementation in SVAT schemes and for testing hypotheses on optimal root behavior in different environments.

With our model, we found a wide range of vertical water uptake profiles even for very similar vertical RLD profiles, which is a result of the individual behavior of each root architecture and its hydraulic parameters. Root architecture becomes more important for the spatial distribution of uptake with time as shown by the increase of confidence bands for the vertical uptake profiles.

The model predictions with the architecture based model aRoot show different behavior than the Feddes Model. The Feddes model distributes and limits root water uptake based on two key properties of the plant or plant community: (1) the root length density profile, and (2) the critical point where water uptake starts to be limited by soil moisture (see $\psi_{3}$ in Fig. 3). Our modeling results with aRoot suggest that both of these properties are not suitable for describing the distribution of real water uptake. While the root length density distribution was similar for all 50 root system realizations, root water uptake profiles differed considerably between individuals. This was especially the case, when assuming relatively low values of root radial resistance (scenario B). Also, transpiration started to be limited at a wide range of bulk water contents, particularly for scenario A, where large root radial resistance was assumed.

Our results suggest that root water uptake behavior might vary greatly between individuals of a particular species. More research is necessary to support this conclusion, and to identify such root properties, which are suitable for describing root water uptake profiles. Also, roots have a complex effect on soil water content and the flow of water through the soil by roots, especially if the interaction between root growth and the surrounding soil is considered. In case of roots clustering in a certain soil volume this might significantly affect the pore space distribution, further impacting on the water holding and soil water movement characteristics.

Acknowledgements. We thank Doris Vetterlein, Andrea Carminati, Mathieu Javaux, Tom Schröder, Vanessa Dunbabin and Jan W. Hopmans for early discussion and comments. This work was kindly supported by Helmholtz Impulse and Networking Fund through Helmholtz Interdisciplinary Graduate School for Environmental Research (HIGRADE).

Edited by: N. Romano and C. Hinz

\section{References}

Amenu, G. G. and Kumar, P.: A model for hydraulic redistribution incorporating coupled soil-root moisture transport, Hydrol. Earth Syst. Sci., 12, 55-74, 2008, http://www.hydrol-earth-syst-sci.net/12/55/2008/.

Clausnitzer, V. and Hopmans, J. W.: Simultaneous modeling of transient three-dimensional root growth and soil water flow, Plant Soil, 164, 299-314, 1994.

de Jong van Lier, Q., Metselaar, K., and van Dam, J. C.: Root water extraction and limiting soil hydraulic conditions estimated by numerical simulation, Vadose Zone J., 5, 1264-1277, doi: 10.2136/vzj2006.0056, 2006.

de Jong van Lier, Q., van Dam, J. C., Metselaar, K., de Jong, R., and Duijnisveld, W. H. M.: Macroscopic Root Water Uptake Distribution Using a Matric Flux Potential Approach, Vadose Zone J., 7, 1065-1078, 2008.

De Willigen, P. and van Noordwijk, M.: Root, Plant. Production and Nutrient Use Efficiency, Ph.D. thesis, Agricultural University, Wageningen, The Netherlands, 1987.

Desborough, C. E.: The impact of root weighting on the response of transpiration to moisture stress in land surface schemes, Mon. Weather Rev., 125(8), 1920-1930, doi:10.1175/ 1520-0493(1997)125〈1920:TIORWO $\rangle$ 2.0.CO;2, 1997.

Doussan, C., Pagès, L., and Vercambre, G.: Modelling of the Hydraulic Architecture of Root Systems: an Integrated Approach to Water Absorption - Model Description, Ann. Bot.-London, 81, 213-223, 1998.

Doussan, C., Pierret, A., Garrigues, E., and Pagès, L.: Water uptake by plant roots: II - Modelling of water transfer in the soil root-system with explicit account of flow within the root system - Comparison with experiments, Plant Soil, 283(1-2), 99-117, doi:10.1007/s11104-004-7904-z, 2006.

Feddes, R., Kowalik, P., Kolinska-Malinka, K., and Zaradny, H.: Simulation of field water uptake by plants using a soil water dependent root extraction function, J. Hydrol., 31, 13-26, 1976.

Feddes, R. A., Hoff, H., Bruen, M., Dawson, T., de Rosnay, P., Dirmeyer, P., Jackson, R. B., Kabat, P., Kleidon, A., Lilly, A., and Pitman, A. J.: Modeling Root Water Uptake in Hydrological and Climate Models, B. Am. Meteorol. Soc., 82, 2797-2809, 2001. 
Gardner, W. R.: Dynamics aspects of water availability to plants, Soil Sci., 89, 63-73, 1960.

Gardner, W. R.: Relation of root distribution to water uptake and availabilty, Agron. J., 56, 41-45, 1964.

Garrigues, E., Doussan, C., and Pierret, A.: Water Uptake by Plant Roots: I - Formation and Propagation of a Water Extraction Front in Mature Root Systems as Evidenced by 2-D Light Transmission Imaging, Plant Soil, 283(1-2), 83-98, doi: 10.1007/s11104-004-7903-0, 2006.

Green, S. R. and Clothier, B.: Root water uptake by kiwifruit vines following partial wetting of the root zone, Plant Soil, 173, 317328, doi:10.1007/BF00011470, 1995.

Jacobsen, B.: Water and phosphate transport to plant roots, Acta Agr. Scand., 24, 55-60, 1974.

Javaux, M., Schröder, T., Vanderborght, J., and Vereecken, H.: Use of a Three-Dimensional Detailed Modeling Approach for Predicting Root Water Uptake, Vadose Zone J., 7, 1079-1089, doi: 10.2136/vzj2007.0115, 2008.

Kolditz, O., Delfs, J.-O., Bürger, C., Beinhorn, M., and Park, C.H.: Numerical analysis of coupled hydrosystems based on an object-oriented compartment approach, J. Hydroinform., 10(3), 227-244, doi:DOI:10.2166/hydro.2008.003, 2008.

Lai, C.-T. and Katul, G.: The dynamic role of root-water uptake in coupling potential to actual transpiration, Adv. Water Resour., 23(4), 427-439, doi:doi:10.1016/S0309-1708(99) 00023-8, 2000.

Levin, A., Shaviv, A., and Indelman, P.: Influence of root resistivity on plant water uptake mechanism, part I: numerical solution, Transport Porous Med., 70, 63-79, doi:10.1007/ s11242-006-9084-1, 2007.

Li, K., Jong, R. D., and Boisvert, J.: An exponential root-wateruptake model with water stress compensation, J. Hydrol., 252, 189-204, 2001.

Pagès, L., Vercambre, G., Drouet, J.-L., Lecompte, F., Collet, C., and Bot, J. L.: Root Typ: a generic model to depict and analyse the root system architecture, Plant Soil, 258, 103-119, 2004.

Schenk, H. and Jackson, R.: The global biogeography of roots, Ecol. Monogr., 72(3), 311-328, 2002.

Schröder, T., Javaux, M., Vanderborght, J., Körfgen, B., and H. Vereecken, H.: Effect of local soil hydraulic conductivity drop using a 3-D root water uptake model, Vadose Zone J., 7, 10891098, doi:10.2136/vzj2007.0114, 2008.

Schröder, T., Javaux, M., Vanderborght, J., Körfgen, B., and H. Vereecken, H.: Implementation of a microscopic soil-root hydraulic conductivity drop function in a 3-D soil-root architecture water transfer model, Vadose Zone J., 8, 783-792, 2009.

Schymanski, S. J., Sivapalan, M., Roderick, M. L., Beringer, J., and Hutley, L. B.: An optimality-based model of the coupled soil moisture and root dynamics, Hydrol. Earth Syst. Sci., 12, 913932, 2008,

http://www.hydrol-earth-syst-sci.net/12/913/2008/.

Sharp, R. E. and Davies, W. J.: Root Growth and Water Uptake by Maize Plants in Drying Soil, http://jxb.oxfordjournals.org/cgi/ content/abstract/36/9/1441, J. Exp. Bot., 36(170), 1441-1456, 1985.
Siqueira, M., Katul, G., and Porporato, A.: Onset of water stress, hysteresis in plant conductance, and hydraulic lift: Scaling soil water dynamics from millimeters to meters, Water Resour. Res., 44, W01432, doi:10.1029/2007WR006094, 2008.

Šimůnek, J., van Genuchten, M. Th., and Šejna, M.: The HYDRUS Software Package for Simulating Two- and Three-dimensional Movement of Water, Heat, and Multiple Solutes in Variablysaturated Media, Technical Manual, Version 1.0, PC Progress, Prague, Czech Republic, p. 241, 2006.

Šimůnek, J., Šejna, M., Saito, H., Sakai, M., and van Genuchten, M. Th.: The HYDRUS-1D Software Package for Simulating the Movement of Water, Heat, and Multiple Solutes in Variably Saturated Media, Version 4.0, HYDRUS Software Series 3, Department of Environmental Sciences, University of California Riverside, Riverside, California, USA, p. 315, 2008.

Šimůnek, J. and Hopmans, J. W.: Modeling compensated root water and nutrient uptake, Ecol. Model., 220, 505-520, doi:10.1016/j. ecolmodel.2008.11.004, 2009.

Sperry, J. S., Stiller, V., and Hacke, U. G.: Xylem hydraulics and the Soil-Plant-Atmosphere Continuum: Opportunities and Unresolved Issues, http://agron.scijournals.org/cgi/content/abstract/ 95/6/1362, Agron. J., , 95(6), 1362-1370, 2003.

Steudle, E.: Water uptake by plant roots: An integration of views, Plant Soil, 226, 45-56, 2000.

Steudle, E. and Peterson, C. A.: How does water get through roots?, http://jxb.oxfordjournals.org/cgi/content/abstract/49/322/775, J. Exp. Bot., 49(322), 775-788, 1998.

Teuling, A. J., Uijlenhoet, R., Hupet, F., and Troch, P. A.: Impact of plant water uptake strategy on soil moisture and evapotranspiration dynamics during drydown, Geophys. Res. Lett., 33, L03401, doi:10.1029/2005GL025019, 2006.

Tuzet, A., Perrier, A., and Leuning, R.: A coupled model of stomatal conductance, photosynthesis and transpiration, Plant Cell Environ., 26, 1097-1116, doi:10.1046/j.1365-3040.2003.01035. $\mathrm{x}, 2003$.

van Genuchten, M.: A closed-form equation for predicting the hydraulic conductivity of unsaturated soils, Soil Sci. Soc. Am. J., 44, 892-898, 1980.

Vrugt, J. A., van Wijk, M. T., Hopmans, J. W., and Šimunek, J.: One-, two-, and three-dimensional root water uptake functions for transient modeling, http://www.agu.org/journals/wr/ v037/i010/2000WR000027/, Water Resour. Res., 37 (10), 2457$2470,2001$.

Wan, C., Yilmaz, I., and Sosebee, R.: Seasonal soil-water availability influences snakeweed root dynamics, J. Arid Environ., 51, 255-264, doi:10.1016/jare.2001.0942, 2002.

Zeng, X., Dai, Y., Dickinson, R., and Shaikh, M.: The role of root distribution for climate simulation over land, http: //www.agu.org/pubs/crossref/1998/1998GL900216.shtml, Geophys. Res. Lett., 25(24), 4533-4536, 1998.

Zwieniecki, M. A., Thompson, M. V., and Holbrook, N. M.: Understanding the Hydraulics of Porous Pipes: Tradeoffs Between Water Uptake and Root Length Utilization, J. Plant Growth Regul., 21, 315-323, doi:10.1007/s00344-003-0008-9, 2003. 